\title{
RENOVACIÓN METODOLÓGICA DE TEORÍAS DE LA COMUNICACIÓN ANTE EL EEES
}

\author{
Adolfo Carratalá ${ }^{1}$ : Universitat deValència. España \\ adolfo.carratala@uv.es
}

\section{RESUMEN}

La implantación del Espacio Europeo de Educación Superior, impulsado en 1999, ha transformado la oferta de títulos de las universidades españolas durante los últimos años. Sin embargo, el nuevo catálogo de grados también debe ir acompañado de una actualización de las estrategias docentes. La renovación metodológica de las materias de carácter más teórico supone un reto que es necesario afrontar con la incorporación de propuestas de aprendizaje colaborativo y con el uso de nuevas tecnologías. En este artículo se analiza el diseño, la aplicación y los resultados que dio el desarrollo de la asignatura de Teorías de la Comunicación según un modelo orientado al aprendizaje de los estudiantes, como exige el nuevo marco educativo.

PALABRAS CLAVE: Teorías de la Comunicación - Metodología - Aprendizaje cooperativo - Nuevas tecnologías - EEES

\section{METHODOLOGICAL UPDATING OF COMMUNICATION THEORIES FACED WITH THE EHEA}

\begin{abstract}
The implementation of the European Higher Education Area, launched in 1999, has transformed the degrees offered by Spanish universities in recent years. However, the new catalog of degrees should also be accompanied by updated teaching strategies. Methodological renewal of the most theoretical subjects is a challenge that must be addressed by incorporating suggestions for collaborative learning and the use of new technologies. This article discusses the design, the application and the results of developing the subject of Communication Theories following a studentcentred learning model as required by the new educational framework.
\end{abstract}

\footnotetext{
${ }^{1}$ Autor correspondiente

Adolfo Carratalá: Docente e Investigador en Formación FPU. Universitat de València. Valencia, España.

Correo: adolfo.carratala@uv.es
} 
KEY WORDS: Communication Theories-Methodology-Cooperative-Learning -New Technologies - EHEA

\section{INTRODUCCIÓN}

La reforma de los planes educativos de los estudios universitarios españoles con ocasión de la implantación del Espacio Europeo de Educación Superior (EEES) exige una profunda revisión de la metodología que durante años ha caracterizado la enseñanza de muchas carreras $y$, en especial, la de aquellas asignaturas que tradicionalmente han sido diseñadas con estrategias docentes basadas en la exposición teórica y la lección magistral. El reto no es sencillo dado que, tal y como indican diversos estudios que se recogen en este artículo, los déficits metodológicos que arrastran algunas materias son especialmente elevados en lo referente a la inclusión de los estudiantes en el proceso de aprendizaje y en la utilización de los nuevos espacios de comunicación digital que ofrecen internet y las redes sociales.

La asignatura de Teorías de la Comunicación, presente en los grados de Periodismo y Comunicación Audiovisual, es una de las que mayores esfuerzos deben realizar para adaptarse a los requisitos que marca la convergencia universitaria europea. La renovación metodológica de esta materia resulta una necesidad dados los tradicionales enfoques que han acompañado su impartición y responde a la transformación que debe experimentar nuestra actividad docente cuando esta tiene que girar en torno al aprendizaje real de los alumnos. El modelo aquí descrito y analizado es una propuesta aplicada en la Universitat de València que, sin ofrecer fórmulas definitivas ni infalibles, intenta avanzar en la superación de los planteamientos conservadores e integrar el aprendizaje cooperativo en el desarrollo del curso.

\section{METODOLOGÍA}

\subsection{De los objetivos a las competencias}

El aprendizaje de los estudiantes ocupa actualmente una posición central en la educación universitaria. Tanto es así que el sistema de créditos anterior, que aludía al trabajo realizado en clase por el profesor, hoy ha dejado paso al llamado crédito europeo (ECTS) que, adaptado al nuevo modelo de formación que se está implantando, hace referencia al volumen de trabajo efectivo del estudiante.

El cambio de modelo educativo debe afectar, por este motivo, a las metodologías empleadas en el ámbito universitario. Estas ya no deben estar tan orientadas hacia el enseñar como hacia el aprender, núcleo hoy de todo el proceso. Este aprendizaje se distingue, además, por ser autónomo (el profesor debe ayudar al alumno a aprender a aprender con cierta independencia y asumiendo un rol activo), para toda la vida (el proceso continuará más allá de la finalización del curso) y cooperativo (el resto de 
compañeros y el mismo docente contribuyen al logro de resultados). Mediante este cambio de orientación, nuestra docencia hoy se dirige no a la consecución de objetivos sino al desarrollo de competencias y, para ello, encuentra dos aliados clave: la introducción de nuevas metodologías y el uso de las TIC.

Respecto a las primeras, cabe decir que "los métodos de enseñanza con participación del alumno, donde la responsabilidad del aprendizaje depende directamente de su actividad, implicación y compromiso son más formativos que meramente informativos, generan aprendizajes más profundos, significativos y duraderos y facilitan la transferencia a contextos más heterogéneos" (Fernández, 2006, p. 42). Por otro lado, las nuevas tecnologías constituyen "un recurso didáctico fundamental para lograr la adaptación al Espacio Europeo de Educación Superior y mejorar, por tanto, la calidad de la enseñanza universitaria" mediante, por ejemplo, el desarrollo de la evaluación continua y formativa y la implementación de proyectos o actividades cooperativas (Guerra et al., 2010, p. 146).

Esta transformación en el proceso de enseñanza-aprendizaje adquiere máxima importancia en aquellas materias que más dificultades o menos interés han encontrado a la hora de introducir en su metodología propuestas innovadoras que fueran más allá de las herramientas tradicionales. La asignatura de Teorías de la Comunicación es un buen ejemplo. Tras un estudio realizado sobre 30 programa académicos de esta materia empleados en el curso 2007/2008 por 28 universidades españolas, los resultados indican que un 95,8\% de ellos incorporaban la clase magistral como metodología docente, convirtiéndose así en la más habitual, mientras que otras propuestas como los trabajos en grupo (12,5\%) o los seminarios $(4,1 \%)$ solo eran introducidos en la actividad del aula por una minoría (García y García, 2009).

Otra investigación sobre las enseñanzas de las Teorías de la Comunicación y de la Información que se imparten en las universidades de Europa y Latinoamérica señala que la gran mayoría de los profesores encuestados $(86,2 \%)$ privilegiaban en su docencia las competencias cognitivas sobre otras como las comportamentales $(9,5 \%)$ o las profesionales $(4,2 \%)$, un desajuste que los responsables del estudio vinculan al marcado carácter teórico de la asignatura y al estado inicial en el que podía encontrarse la renovación metodológica de los planes de estudio europeos en el momento de realizar los cuestionarios. Este mismo proyecto desveló que muchos profesores aún rigen sus métodos por criterios conservadores: el 73,8\% aseguró que sus clases teóricas abarcaban más de la mitad del curso académico y el 32,1\% reconocía no dar ningún valor en la nota final a la participación en clase (Lozano y Vicente, 2010).

Por este motivo, son diversos los autores que se han preocupado por reflexionar de qué modo puede adecuarse al nuevo modelo educativo la asignatura de Teorías de la Comunicación. En primer lugar, resulta clave tener en cuenta cómo ha de trasladarse el foco desde los antiguos objetivos a las competencias cuyo desarrollo ahora debe orientar nuestra actividad docente. Así, la materia que nos ocupa puede facilitar que los alumnos adquieran competencias relativas a la comunicación y a la creatividad, 
interpersonales, valores éticos y emocionales y también aquellas vinculadas con la comprensión del entorno y con el desarrollo del autoaprendizaje (Estrada y Rodrigo, 2007).

Para lograr que los estudiantes alcancen las competencias que nuestra asignatura permite adquirir, sería necesario renovar las estrategias docentes e incorporar "metodologías de enseñanza y sistemas dinámicos de prácticas y de carácter investigador que completaran a las clases magistrales" haciendo uso de "seminarios sobre temáticas concretas con grupos reducidos" y otorgándole mayor peso a "la participación en foros, weblogs y demás soportes web" (García y García, 2009, p. 289). En este sentido, el fomento de la lógica de la red y la construcción de redes sociales y comunicativas permitirían romper con "los modelos jerárquicos, verticales y unidireccionales" que aun dominan muchos espacios educativos y, así, acercarnos al lenguaje de los vínculos y la interacción, tan familiares para las jóvenes generaciones (Marí, 2006, p. 116).

Siguiendo, entre otras, estas indicaciones, la apuesta por la innovación metodológica ha sido una de las prioridades en el desarrollo de la asignatura de Teorías de la Comunicación, de 6 ECTS, que se imparte en el 2do curso del Grado en Periodismo de la Universitat de València.

\section{ANÁLISIS Y DISCUSIÓN}

\subsection{Aprendizaje interdependiente, responsabilidad de todos}

Como hemos avanzado, el aprendizaje cooperativo o colaborativo es uno de los faros que debe guiar la acción docente en la educación universitaria que se está implantando tras la convergencia europea. Este tipo de metodología permite incorporar dinámicas de participación que ayudan a hacer del proceso de aprendizaje una experiencia compartida en la que los alumnos juegan un papel destacado. Su acción resulta imprescindible no solo para el avance de las sesiones sino para que estas resulten productivas y, por consiguiente, ellos logren adquirir las competencias previstas en la planificación del curso.

De este modo, coincidimos con que la formación de competencias exige que el estudiante se responsabilice de su propio aprendizaje y hace necesaria "la capacidad para aprender con los otros de manera cooperativa, fomentando el intercambio de ideas, opiniones, puntos de vista" (Fernández, 2006, p. 43). Así, esta dinámica contribuirá al desarrollo de una estructura de colaboración e interdependencia de la que pueden resultar beneficiados todos los participantes en la dinámica de aprendizaje.

Las virtudes de este tipo de metodología han sido puestas de manifiesto por numerosas investigaciones $\mathrm{y}$, entre otras, podríamos destacar que promueve la implicación activa, incrementa los niveles de aprendizaje, reduce el abandono, desarrolla el razonamiento crítico, la capacidad de expresión oral, prepara para ser ciudadanos y para desenvolverse en el mundo laboral (Valero-García, 2010). 
La interacción entre los estudiantes resulta clave para que pueda llevarse a cabo la cooperación que les conducirá al logro de las competencias mencionadas. Por un lado, la interacción cara a cara debe garantizarse en las horas de clase, de forma física, aunque los grupos de universitarios también puedan llevar a cabo sus trabajos fuera del aula. Por otro, la introducción de las TIC también contribuye a impulsar este tipo de metodología, a través de la interacción mediada tecnológicamente, a la que los jóvenes actuales se encuentran tan acostumbrados. Para ello, el docente debe ofrecer espacios y plataformas que los alumnos puedan aprovechar para llevar a cabo la colaboración virtual.

Para favorecer la cooperación presencial, en el curso cuyas experiencias se analizan en este estudio, el grupo de alumnos, que contaba con 74 estudiantes matriculados, fue dividido en dos subgrupos durante las sesiones prácticas con el objetivo de facilitar la participación y la implicación de todos y todas en los diferentes seminarios presenciales, que ocuparían el 50\% del total de las clases programadas, reduciendo para ello el número de sesiones expositivas

\subsection{Propuestas de cooperación presencial y virtual}

La introducción de una metodología docente que permitiera dar con los propósitos descritos anteriormente exigía dotar a la asignatura de Teorías de la Comunicación de un enfoque en el que destacara la participación de los alumnos y alumnas. La interacción entre ellos, entre ellos y el profesor e, incluso, entre ellos y otros usuarios de los espacios en los que se desarrollaran algunas prácticas adquiría una importancia crucial en esta nueva propuesta.

Para unos jóvenes que se están formando como profesionales de la comunicación, la inclusión de estrategias pedagógicas que giraran en torno a ella contribuiría sin ninguna duda al desarrollo de muchas de las competencias contempladas en el plan de estudios. Los espacios para ejecutarlas serían la clase física tradicional y el virtual, propiciado por las nuevas tecnologías e internet. Su puesta en marcha debía, además, contar con peso en el sistema de evaluación para, así, reflejar también en ese apartado que la renovación metodológica debe afectar a toda la propuesta didáctica, sobre todo en materias que destacan por ser eminentemente teóricas. De este modo, las distintas tareas que se articulan bajo la lógica de la cooperación y la interacción supondrían hasta un $50 \%$ de la calificación final.

\subsubsection{Seminarios y talleres en el aula}

Las sesiones presenciales de carácter práctico fueron programadas de tal modo que acompañaban a cada sesión de carácter expositivo, permitiendo profundizar y complementar las cuestiones tratadas en ellas. Estos encuentros fueron diseñados a partir de los materiales que dotarían de contenido cada sesión. Así, diferenciamos entre seminarios basados en textos y los que iban a ser articulados a partir de la visualización o escucha conjunta de determinados documentos audiovisuales 
La opción de integrar seminarios en la programación de la asignatura, además de responder a las recomendaciones de estudios precedentes, adquiere toda su importancia en un planteamiento metodológico que sitúa al estudiante en el centro del proceso de aprendizaje. Articular seminarios abiertos supone confiar en la discusión conjunta y en la multiplicidad de perspectivas como vías de entendimiento y desarrollo de competencias. Así, estas experiencias, cuyo resultado no debe estar predeterminado, tienen como propósito que "los estudiantes profundicen su comprensión de algo que ya han examinado previamente" y que se convenzan "de que serán ellos los que hagan el trabajo duro de la indagación" (Finkel, 2008, p. 78).

Las experiencias aquí recogidas ilustran algunas de las propuestas ideadas bajo el principio de trabajo cooperativo. Entre las sesiones prácticas desarrolladas a partir de textos escritos, siempre facilitados a los estudiantes con el tiempo necesario para que acudan a la sesión práctica con la lectura realizada, destacaremos tres. Para cada una de ellas, se optó por una actividad de aprendizaje específica, en función de las características y las posibilidades del material de trabajo: "Comunicación de masas, gustos populares y acción social organizada" - Puzle de Aronson

El trabajo sobre este conocido texto de Paul Felix Lazarsfeld y Robert King Merton se realizó mediante la metodología del Puzle de Aronson, que consiste en dividir el material de trabajo en tres o cuatro partes razonablemente independientes y establecer una técnica de análisis cooperativa e interactiva. En este caso, el documento quedó dividido en cuatro partes: Introducción, funciones sociales de los mass-media, estructura de la propiedad y control de los mass-media y propaganda para objetivos sociales. Una vez diferenciadas estas secciones, se pidió a los estudiantes que formaran grupos de cuatro y que, tras adjudicarse un número, repasaran de forma concentrada el fragmento que correspondía a su responsabilidad.

Tras ello, hubieron de reunirse por "grupos de expertos" con el objetivo de debatir en profundidad y resolver dudas de cada una de las partes con los compañeros que también se habían especializado en ellas. Por último, cada alumno volvía a su grupo original para explicar a sus compañeros y compañeras las conclusiones principales del fragmento que había trabajado y, a la vez, recibir similar información del resto del texto por los otros. Finalmente, entre todos se llevó a cabo una discusión general abierta sobre los aspectos que habían resultado más relevantes durante el trabajo colaborativo.

“La obra de arte en la época de su reproductibilidad técnica” - Seminario y debate.

La sesión sobre este ensayo de Walter Benjamin quedó articulada en dos debates entre posiciones antagónicas que aparecen en dicho texto. El grupo de estudiantes quedó dividido en cuatro subgrupos, con el objetivo de que, en cada debate, pudiera haber alumnos protagonizándolo y otros observándolo y participando desde fuera. Los temas propuestos fueron: ¿El aura de la obra de arte es un elemento positivo que debería conservarse o un aspecto negativo para lograr su popularización? y ¿La relación entre el arte y la política es nociva o el arte puede ayudar a la emancipación 
de los individuos si es politizado? La discusión, que se inició orientada bajo estas cuestiones y con argumentos elaborados a partir de las ideas presentes en el ensayo, fue abriéndose poco a poco a inquietudes que la interacción entre los estudiantes fue despertando.

"Treinta años de economía política de la comunicación: las aportaciones de Herbert I. Schiller" - Tabú

Dado que el contenido de este texto, de Ana I. Segovia, no resultaba tan complejo como los anteriores, el enfoque del seminario adoptó un planteamiento más lúdico, sin dejar de abordar cuestiones de interés. Así, se optó por utilizar la técnica del conocido juego de mesa 'tabú', en el que los participantes deben explicar una serie de conceptos a sus compañeros sin emplear un conjunto de palabras prohibidas. Por tanto, por un lado era necesario reflexionar y razonar para dar una definición propia del término escogido al azar entre un conjunto de fichas y, por otro, hacerse entender y elaborar un mensaje claro y eficiente. La actividad se amplió de tal manera que la persona que acertaba el concepto que se estaba explicando debía volver a definirlo pero, esa vez sí, incluyendo en su exposición todas las palabras obligatoriamente excluidas en la primera. En total, se elaboraron diez fichas con cinco palabras prohibidas en cada una. Como ejemplo, la tarjeta de "Imperialismo cultural" incluía como voces censuradas: EEUU, comunicación, colonialismo, empresas y dominio.

En el resto de seminarios, los materiales utilizados para iniciar el debate y la reflexión colectiva fueron de carácter audiovisual. Exponemos también tres ejemplos

\section{Emisión radiofónica "La guerra de los mundos"}

El grupo de estudiantes escuchó la versión que Radio Nacional emitió del programa conducido en 1938 por Orson Welles, ampliamente conocido por haber ocasionado en su día importantes efectos en la población, que creyó que la narración de una supuesta invasión del planeta por parte de marcianos informaba de hechos reales. Los alumnos pudieron, así, situarse frente al mensaje que hace decenas de años motivó el pánico en una sociedad todavía muy crédula respecto a lo que se afirmaba desde los medios de comunicación masivos. Tras la escucha, una puesta común entre todos los estudiantes permitió reflexionar sobre los elementos de la emisión que pudieron influir en su recepción como un mensaje veraz y sobre los motivos que hoy dificultarían que se diera un fenómeno similar.

\section{Visionado de la película "La cortina de humo"}

Los seminarios planteados a partir de la reproducción de películas también motivaron el interés y la participación de los estudiantes. En este caso, el filme señalado, que muestra cómo el gobierno estadounidense es capaz de organizar espectaculares campañas de propaganda con el objetivo de construir y crear falsos acontecimientos para ocultar otros que sí existen pero que afectan a sus intereses partidistas y electorales, permitió introducir un debate abierto en torno a cuestiones 
como el sistema político, la opinión pública, la acción mediática y el construccionismo. Estas cuestiones fueron sugeridas por el docente ya que, pese a que un seminario siempre debe encontrarse abierto a un resultado imprevisible, el profesor puede ayudar actuando como faro y así "mejorar la posibilidad de que el aprendizaje ocurra influyendo en el desarrollo de la conversación" (Finkel, 2008, p. 91).

\section{Visionado de documentales}

Del mismo modo que hemos comentado con la película, la discusión colectiva, abierta a todos y todas, a partir de la reproducción de documentales vinculados a las cuestiones incluidas en el programa de la guía docente, como "Vender la guerra" o "Manufacturing consent", permitió profundizar en los temas previamente abordados de un modo más expositivo. En este caso, dado que los contenidos de ambas producciones hacen referencia a acontecimientos reales y a la cobertura que de ellos llevaron a cabo los medios de comunicación, la motivación con la que los estudiantes intervinieron y el nivel de aportaciones realizadas fueron realmente notables, resultando muy limitado el tiempo de la sesión para dar cabida a todas las opiniones que pretendían expresarse, tal y como ocurre en los seminarios realmente abiertos, donde "la conversación terminará porque el tiempo se habrá acabado, no porque se haya llegado a un final" (Finkel, 2008, p. 90).

Por último, mencionaremos la metodología empleada en las últimas tres sesiones del curso, en las que los alumnos y alumnas volvieron a ser los responsables de su desarrollo. Durante esas clases presenciales, los estudiantes debían realizar exposiciones que conjugaban el 'role play' con el panel científico, dado que debían presentarse como equipos de investigación e ilustrar creativamente las posibilidades de aplicación actual de las teorías principales analizadas a lo largo del cuatrimestre. Los compañeros que no participaban en la exposición tenían una doble tarea: intervenir mediante preguntas y evaluar al grupo que exponía con la ayuda de una rúbrica facilitada por el docente en la que debían considerarse tres competencias (presentación oral, integración de la información y trabajo en grupo) y cuatro posibles niveles en las que situarlas (todavía no competente, aceptable, competente y muy competente). De este modo, la evaluación entre compañeros permitía asumir los criterios considerados y fomentar la reflexión y la capacidad de ser crítico con el trabajo de los otros y también con el propio.

\subsection{La aplicación de las TIC}

El empleo de las posibilidades que ofrecen las nuevas tecnologías mejora la calidad de la docencia ya que, por un lado, permite superar las limitaciones espaciotemporales de las relaciones presenciales y, por otro, facilita que los universitarios adquieran destrezas en el uso de unas herramientas que formarán, con mucha probabilidad, parte de su futura labor profesional (Martín, 2010). Sin embargo, la contribución de la tecnología a la programación docente debe resultar complementaria $\mathrm{y}$, por lo tanto, no debe ser contemplada ni como la única válida ni como la sustituta de otras metodologías aue siquen encontrando su meior ámbito de desarrollo en la 
clase física, pues como han indicado algunos estudios, pese a que los estudiantes ven positivo el desarrollo de comunidades educativas en línea y de estudios online, consideran que "no debería nunca acabar con las clases presenciales y con el modelo tradicional de enseñanza" (Santín et al., 2011, p. 748)

Así, el empleo de herramientas virtuales se introdujo como propuesta para acometer parcialmente los contenidos prácticos de la materia. En este sentido, destacan tres iniciativas diferentes pero con un aspecto común: todas se desarrollaron en internet.

La primera de ellas hizo uso de una de las diversas aplicaciones en línea que permiten el desarrollo de espacios wiki para el trabajo colaborativo. El objetivo era la puesta en marcha de un glosario que recogiera los conceptos y autores básicos que fueran a discutirse a lo largo del curso. Para ello, se creó una página dedicada a la asignatura y accesible desde la dirección web http:/ / teoriesdelacomunicacio.wikispaces.com/. Los contenidos, pese a ser públicos $\mathrm{y}$ visibles para cualquier internauta, se encuentran protegidos $\mathrm{y}$, por lo tanto, solo pueden ser editados por aquellos participantes que hayan sido autorizados por el creador del espacio.

El listado de entradas que habían de ser trabajadas, agrupadas bajo los distintos apartados que constituían el programa de la asignatura, fue propuesto por el profesor y, cada estudiante, pudo reservar el término preferido accediendo a la wiki tras haber registrado su propio nombre de usuario. En total, al finalizar el curso, de las 73 propuestas que incluía el glosario, habían sido elaboradas 65; es decir, el 89\% del total. En cada entrada, el autor debía desarrollar los aspectos más importantes sobre el concepto escogido, citar las fuentes de información de las que obtuvo los datos, insertar elementos multimedia si resultaba posible e incluir enlaces con otros documentos relacionados ubicados en otras páginas de internet pero, también, con algunos de los términos trabajados por sus compañeros con los que el concepto propio pudiera guardar relación.

Este último aspecto permitió que, aunque el trabajo principal fuera de carácter autónomo y creativo, cada alumno tuviera también que contar con la tarea de los demás para completar la suya propia, generando de ese modo cooperación. Otro aspecto que subraya la responsabilidad compartida que para los estudiantes suponía esta tarea lo manifiesta el que fuera presentada por el profesor y asumida por el grupo no solo como una práctica sujeta a evaluación sino como una herramienta de ayuda al estudio que los alumnos desarrollaban por y para sí mismos, con el objetivo de resultar útil durante la preparación individual de la asignatura.

El segundo espacio de trabajo en línea se desarrolló dentro del Aula Virtual que la Universitat de València activa a docentes y estudiantes para aprovechar las posibilidades que ofrece la plataforma en las distintas asignaturas. En este curso, se optó por poner en marcha la aplicación de los foros y, así, poder llevar a cabo debates vinculados con los contenidos de la asignatura que no se vieran limitados por la 
duración de las sesiones presenciales, de dos horas cada una. En este caso, tanto el acceso como la participación quedan limitados a los alumnos matriculados en la materia.

Finalmente, durante los cuatro meses que duró el curso, el foro acogió 20 temas de debate, cuatro de los cuales fueron abiertos por el responsable de la materia mientras que los 16 restantes surgieron por iniciativa propia de los matriculados en la asignatura. Pese a que la previsión inicial del docente era la apertura de un nuevo debate cada dos semanas, el surgimiento de discusiones sugeridas por los alumnos limitó las conversaciones iniciadas por él para evitar dispersar la atención de los estudiantes y potenciar, en cambio, sus intereses dinamizando los temas propuestos.

Prácticamente el 50\% de los debates, nueve de las 20 discusiones, recogieron más de diez intervenciones durante el desarrollo de la conversación, alcanzando, por ejemplo, las 25 opiniones en uno de los debates, que por su vínculo a la actualidad y por su carácter controvertido (el tratamiento mediático sobre la muerte de Bin Laden), resultó ser el que más participación despertó entre los estudiantes. En total, los diferentes hilos de discusión generaron 179 aportaciones entre los meses de febrero y mayo. Por último, cabe destacar que algunos de los debates lograron extenderse durante gran parte del cuatrimestre, de modo que ciertos temas llegaron a generar reacciones de los alumnos durante dos meses.

Por último, la tercera fórmula de interacción online entre los alumnos se ejecutó en la red social Facebook. En este sentido, coincidimos con la importancia que algunos autores atribuyen a la inclusión de estas aplicaciones en la enseñanza universitaria: permiten ir más allá de las horas de clase, implican una interacción activa y constante entre los alumnos y profesores, cumplen con lo previsto en el marco del Espacio Europeo de Educación Superior (EEES) y convierten a la enseñanza en un proceso cíclico, en el que las clases presenciales pueden seguir en la red social y las contribuciones que en esta se desarrollan pueden aprovecharse para iniciar la siguiente sesión en el aula física (Túñez y Sixto, 2011). Estos motivos adquieren mayor peso aún entre estudiantes de Periodismo y Comunicación, pues su participación en redes sociales se encuentra en incremento, sobre todo con fines comunicativos y relacionales según indican algunas investigaciones (Martínez y Torrado, 2011), su atracción por los temas de actualidad se presume máxima y, además, deben desarrollar durante su formación las competencias necesarias como para participar con solvencia y rigor en el debate público, en un momento en el que el ejercicio de la opinión está siendo tan pervertido por numerosos medios de comunicación.

Así, los alumnos y alumnas que se sumaron a la página creada en esta red social bajo el nombre de "Teories de la Comunicació - PE -UV" tuvieron la opción de, no solo compartir y opinar sobre los contenidos de actualidad que encontraran relevantes en el marco de la asignatura, sino también de comentar noticias e informaciones en un espacio abierto a cualquier internauta registrado en Facebook 
Desde su puesta en marcha hasta la finalización del curso, la página logró ser seguida por 44 usuarios, la gran mayoría estudiantes de la asignatura. En esos cuatro meses, el espacio contó con 198 publicaciones, que casi siempre consistían en enlazar una noticia que pudiera ser observada y analizada bajo los presupuestos y fundamentos teóricos discutidos y trabajados en el aula. Hasta 103 publicaciones fueron realizadas por el profesor responsable de la materia mientras que las 95 restantes fueron aportadas por los demás usuarios y usuarias. En numerosas ocasiones, estas contribuciones generaban comentarios que, aunque no lograban ni la extensión ni el desarrollo de los debates localizados en el foro del Aula Virtual, sí que conseguían despertar la curiosidad de otros compañeros que, a su vez, interactuaban enriqueciendo las conversaciones con sus puntos de vista. Algunos de las publicaciones acogieron hasta nueve opiniones sobre su contenido.

Según las estadísticas que la misma red social facilita sobre la página creada, podemos indicar que en ella se dieron 198 interacciones, 79 de las cuales, un 40\%, tomaron la forma de comentarios de los usuarios mientras que el $60 \%$ restante se limitó a la mínima interacción que implica el que el internauta pulse sobre la opción "me gusta" que acompaña cualquier nuevo enlace u opinión publicados en la página. El número de veces que los usuarios accedieron a la página fue variable a lo largo de estos meses, aunque hubo jornadas que se lograron recibir hasta 51 visitas.

\section{CONCLUSIONES}

El modelo de renovación metodológica expuesto en este artículo introduce modificaciones en el desarrollo tradicional que en muchas ocasiones ha caracterizado la enseñanza de Teorías de la Comunicación en las licenciaturas de Periodismo. Con el objetivo de integrar algunas de las propuestas sugeridas por los estudios que previamente habían analizado el estado de los programas docentes de esta materia en las universidades españolas, la propuesta aquí analizada señala algunas de las opciones al alcance de los profesores. Así, pese a que este planteamiento continuará evolucionando y adaptándose en función de los resultados logrados, significa un primer avance hacia los cambios que deben ir asociados a la convergencia educativa europea en la que se han inmerso nuestros estudios superiores.

El aprendizaje cooperativo supone, de este modo, una gran oportunidad para los grados en Periodismo. La comunicación y la interacción que se alcanza con esta metodología favorecen el protagonismo de los estudiantes en su propio proceso de aprendizaje, que afrontan corresponsablemente. Los resultados obtenidos durante el curso señalan que los alumnos y alumnas encuentran estimulante contar con un espacio virtual que permita superar las limitaciones del aula tradicional y que, además, se articule en los entornos con los que se encuentran familiarizados.

La finalidad de este nuevo enfoque metodológico, el desarrollo de competencias, parece más próxima en la medida en que el grupo participa y asume un rol principal en el transcurso de las sesiones. La colaboración que los alumnos desarrollan de manera interdependiente, $\mathrm{v}$ el trabaio aue realizan en las redes de comunicación que 
se abren con esta actualización de la asignatura, les permiten aprovecharla no solo como un proceso conjunto de aprendizaje sino también como un medio de profesionalización en el que ejercitar sus destrezas periodísticas.

\section{REFERENCIAS}

Estrada, A.\& Rodrigo, M.(2007). El reto de la docencia de Teoría de la Comunicación on line y presencial en el marco del EEES . Revista Estudos em Comunicação, (1), 332361.

Fernández March, A. (2006). Metodologías activas para la formación de competencias. Revista Educatio siglo XXI, (24), 35-56.

Finkel, D. (2008): Dar clase con la boca cerrada. Valencia: Publicacions de la Universitat de València..

García Avilés, J. A. \& García Jiménez, L. (2009). La enseñanza de Teorías de la Comunicación en España: análisis y reflexión ante la Convergencia de Bolonia. Revista Zer, 14, (27), 271-293.

Guerra, S.; González, N. \& García, R. (2010). Utilización de las TIC por el profesorado universitario como recurso didáctico. Revista Comunicar, XVIII, (35), 141-148.

Lozano Ascencio, C. \& Vicente Mariño, M. (2010). La enseñanza universitaria de las Teorías de la Comunicación en Europa y América Latina. Revista Latina de Comunicación Social, (65). Recuperado el 10 de Julio de 2011, de http://www.revistalatinacs.org/10/art2/898_URJC/20_Lozano.html.

Marí Sáez, V. M. (2006). Jóvenes, tecnologías y el lenguaje de los vínculos. Revista Comunicar, (27), 113-116.

Martín Llaguno, M. (2010). Una enseñanza de la Teoría de la Comunicación basada en el diseño modular: fortalezas y debilidades. Revista Diálogos de la Comunicación, ( 80 de abril-junio). Recuperado el 28 de Agosto de 2011, de http:// www.dialogosfelafacs.net/revista/articulos-resultado.php?ed=80\&id=139.

Martínez Rodríguez, L.\& Torrado Morales, S. (2011). Uso de las redes sociales como fuente de información de los futuros profesionales de la comunicación: ¿ilusión o realidad? Revista Trípodos, (número extra), 359-366.

Santín Durán, M.; Mathieu, G. \& Gómez Escalonilla, G. (2011). Una universidad sin profesores. Estudio prospectivo sobre los campos virtuales. Revista Trípodos, (número extra), 741-749. 
Túñez López, M. \& Sixto García, J. (2011). Social networks in the university teaching. En Ciastellardi, M. \& et al (Eds): McLuhan Galaxy Conference understanding media, today. Barcelona: Editorial Universidad Oberta de Catalunya, pp 487-496.

Valero García, M. (2010). Por qué aprendizaje cooperativo?. GIAC: Grupo de Interés en Aprendizaje Cooperativo. Recuperado el 28 de Agosto de 2011, de http://giac.upc.es/pag/giac_cas/giac_por_que.htm.

\section{Adolfo Carratalá}

Licenciado y DEA en Periodismo por la Universitat de València, Adolfo Carratalá trabaja desde 2008 como investigador y docente en formación bajo al programa FPU del Ministerio de Educación. En 2011 recibió el Young Scholars Excellence Award por su contribución al VI Congreso Internacional de Comunicación y Realidad 'Life without media'. Realizó una estancia de investigación en la University of Glasgow durante 2010 y otra en el Boston College durante 2011, ambas financiadas por el programa ministerial. Como profesor en formación, asume desde 2010 la docencia de Teorías de la Comunicación en el Grado de Periodismo de la Universitat de València. 